\title{
Alloplasmic lines (Hordeum vulgare)-Triticum aestivum with complete cytonuclear compatibility are the sources of introgression DH lines for wheat breeding
}

\author{
Pershina L.A. ${ }^{1,2 *}$, Trubacheeva N.V. ${ }^{1,2}$, Belan I.A. ${ }^{3}$, Rosseeva L.P. ${ }^{3}$ \\ ${ }^{1}$ Institute of Cytology and Genetics, SB RAS, Novosibirsk, Russia \\ ${ }^{2}$ Kurchatov Genomic Center of the Institute of Cytology and Genetics, SB RAS, Novosibirsk, Russia \\ ${ }^{3}$ Omsk Agricultural Scientific Center, Omsk, Russia \\ *pershina@bionet.nsc.ru
}

\begin{abstract}
Alloplasmic lines are considered as an additional source of diversity of cultivated plants, since when the cytoplasm is replaced by an alien one, new intergenomic interactions arise, leading to variability of adaptive and agronomically important traits. In our works, using the alloplasmic recombinant lines (H. vulgare)-T. aestivum, it was shown that only lines carrying alien cytoplasm with a complete process of cytonuclear coadaptation can be used in breeding. In such lines, cytonuclear compatibility was not disturbed under in vitro conditions, as well as during introgression of alien germplasm into the nuclear genome. Based on the analysis of the regions of mitochondrial and chloroplast DNA, as well as the influence of individual chromosomes on the fertility of alloplasmic lines, markers were identified that allow us to distinguish between alloplasmic recombinant lines with incomplete and complete cytonuclear compatibility. It has been shown for the first time that alloplasmic lines (H.vulgare)-T.aestivum with incomplete cytonuclear compatibility are suitable models for identifying chromosomes carrying restorer fertility genes $(R f)$ of bread wheat $T$. aestivum with $H$. vulgare cytoplasm. On the basis of alloplasmic recombinant introgression lines $(H$. vulgare)-T. aestivum with complete cytonuclear compatibility, DH lines were obtained, which were successfully used in breeding to obtain new promising breeding material [1] and commercial varieties of spring bread wheat. Examples of such varieties for which patents have been obtained: Sigma, Uralosibirskaya 2, Ishmskaya 11 [2] and the Sakmara variety is being tested in the State Variety Trial.

Acknowledgements: This work was supported by project No. 0259-2021-0018, RFBR grant No. 20-016-00196 and the Kurchatov Genomic Center of the Institute of Cytology and Genetics, SB RAS according to the agreement with the Ministry of Education and Science RF, No. 075-15-2019-1662.
\end{abstract}

\section{References}

1 Pershina L., Trubacheeva N., Badaeva E., Belan I., Rosseeva L. Study of androgenic plant families of alloplasmic introgression lines (H. vulgare) $T$. aestivum and the use of sister DH lines in breeding. Plants (Basel). 2020;9:764-816. DOI: 10.3390/plants9060764.

2 Pershina L.A., Belova L.I., Trubacheeva N.V., Osadchaya T.S., Shumny V.K., Belan I.A., Rosseeva L.P., Nemchenko V.V., Abakumov S.N. Alloplasmic recombinant lines (H. vulgare)T. aestivum with 1RS.1BL translocation: initial genotypes for production of common wheat varieties. Vavilov J. Genet. Breed. 2018;22:544-552. DOI: 10.18699/VJ18.393. 